A. Pramesh Rao, G. Swarup and Gopal-Krishna, eds.

\title{
Low Frequency Catalogues of the CATS Database
}

\author{
O.V. Verkhodanov, S.A. Trushkin \\ Special Astrophysical Observatory, Nizhnij Arkhyz, Russia, 369167 \\ H. Andernach \\ Depto. de Astronomía, Apdo. Postal 144, Univ. Guanajuato, \\ Guanajuato, Mexico
}

\begin{abstract}
We describe the most complete, publicly accessible database of radio sources, CATS ("astrophysical CATalogues Support system"). It contains more than 300 catalogues of objects detected in various (mostly radio) wavelength ranges. Apart from catalogues drawn from the most important existing surveys and compilations (e.g. NVSS, FIRST, GB6, IRAS, ROSAT, PGC, MCG), CATS includes the largest low-frequency catalogues published so far: UTR, Culgoora, Cambridge, Miyun, WENSS, TXS, Bologna and Molonglo etc., CATS allows one to operate with these, and to draw samples of objects for the study of a large variety of astrophysical problems. Some modes of access are described here.
\end{abstract}

The creation of the CATS database (http://cats.sao.ru) was motivated on the one hand by the measurements of radio continuum spectra with the RATAN600 telescope (by two of us, O.V. and S.T.), and, on the other hand, by the activity of the third author (H.A.) in collecting the astrophysical catalogues. CATS became operational in 1996.

At present, 140 of the 300 catalogues available in CATS include data at frequencies less or equal to $1400 \mathrm{MHz}$. The largest or the most important of them are all the Cambridge catalogues from $3 \mathrm{C}$ to $8 \mathrm{C}(38,151,178,408 \mathrm{MHz})$, the VLA FIRST and NVSS catalogues at $1400 \mathrm{MHz}$, the $2^{\text {nd }}$ and $3^{\text {rd }}$ Bologna surveys at $408 \mathrm{MHz}$, the Westerbork catalogues at $325 \mathrm{MHz}$, the Texas catalogue at $365 \mathrm{MHz}$, the Parkes catalogues at several frequencies, the GB catalogues, the RATAN-600 catalogues. There are further dedicated catalogues of radio sources, like e.g. those in clusters of galaxies, by Slee, Owen or Reynolds. The CATS database includes a large number of Galactic radio sources, supernova remnants and their maps, observed with the RATAN-600, Effelsberg, Ooty, VLA and other telescopes. The main objective of the CATS database is to help a user to operate with a large number of astrophysical catalogues which have been published in different formats, each one including a somewhat different set of observational parameters.

Among the options accessible to CATS users we mention:

- Request short descriptions of each catalogue, or print a list of catalogues covering the required sky areas. 
- Select objects from one or more catalogues by coordinate, flux, spectral index, frequency, etc.

- Select objects from one or more catalogues for many sky patches defined by position and size (e.g. for cross-ID with other catalogues).

- Display radio spectra of selected sources.

Among other applications, CATS may be used for object identification, source counts in different wavelength ranges, or the study of statistical properties of different source populations.

Access to CATS is possible in three different ways:

(1) http://cats.sao.ru (to see the various options and to search for catalogues)

(2) e-mail: cats@sao.ru (an empty email delivers a help file)

(3) ftp://cats.sao.ru (anonymous FTP, e.g. to copy entire catalogues)

Batch requests to extract objects from large lists of sky regions are supported via e-mail. HTTP and e-mail access permits the user to obtain informtaion from catalogues in selected areas, and to search for cross-identifications of entries in CATS with user-specified input lists.

Here are some simple examples (input formats) of e-mail requests which are very convenient for a slow network transfer:

1. Search an area defined by limits in equatorial coordinates for objects drawn from all radio catalogues, with a flux density above $500 \mathrm{mJy}$ :

mail cats@sao.ru (with the following lines in the body of the email)

cats select

ra $\min =12: 30 \max =12: 40: 15$. dec $>0<5^{\prime} 46^{\prime \prime}$

catalogs $r$ epoch $=1950$ flux $>500$

cats end

2. Request a list of objects from all catalogues in CATS, within an elliptical area of diameter $60 "$ (RA) by $2^{\prime}$ (DEC) around three positions in the sky (equinox B1950) :

mail cats@sao.ru

cats match catalogs a

window box $x=60$ " $y=2$ '

sources :

$\begin{array}{lll}\text { s1 } 02: 02: 00 & +31: 23: 16 & 1950 \\ \text { s2 } 02: 23: 10 & 00: 03: 00 & 1950 \\ \text { s3 } 21: 26: 33.9 & -18: 34: 33.0 & 1950 \\ \text { cats end } & & \end{array}$

Standard formats of output of CATS are either an ASCII table, or the "native" format of the catalogue as published, or a FITS table-like format. The default output format is the ASCII table, containing the source name, its equatorial coordinates with errors, observing frequency and flux density with errors. Radio source spectra may be prepared with CATS from certain multifrequency catalogues and can be retrieved in PostScript format.

Acknowledgments. We are grateful to Vladimir Chernenkov for his help with the software design and for organizing the remote access systems for CATS. 